Int. Pharmacopsychiat. 17: 303-313 (1982)

\title{
Tranquillizer Effects in an Experimental Analog of Group Psychotherapy
}

\author{
Hans-Peter Krüger ${ }^{\mathrm{a}}$, Ralf Kohnen ${ }^{\mathrm{b}}$ \\ a University of Würzburg, and ${ }^{b}$ University of Erlangen-Nürnberg, FRG
}

Key Words. Tranquillizer - Social stress situations - Group dynamics $\cdot$ Healthy subjects - Differential effects of stressors - Anxiolysis - Telemetry · Activation

Abstract. In an experimental analog of group psychotherapy situations, the effects of two dosages of a tranquillizing agent (prazepam) are compared with those of placebo treatment. Pulse frequency and psychic strain on 24 healthy young volunteers was measured in different social interactions. Variations of social situations induced great changes both in physiological and in psychological measures. The drug acted differentially as to sex and dosage, but showed no situation-specific action. These and further results indicated that group dynamics are an efficient tool in proving drug effects.

\section{Introduction}

A crucial problem in evaluating tranquillizer effects in healthy subjects is to induce a high degree of activation. Only under these conditions can the anxiolytic effects of the drug be demonstrated in experimental studies. The well-known 'classical' methods are white noise at high intensity, cold pressure test, competitive performance tasks and so on. All these methods have one characteristic in common: they are (in the experience of the subjects) 'induced by the experimenter'. In fact they represent an asymmetric interaction between experimenter and subject, perhaps sometimes even a competition between the two, the former trying to get his subject stressed, the latter trying to resist. Thus the stressing treatment often leads to artificial laboratory situations. As a result these experimental settings lack external 
or ecological validity for everyday life, a fact which is often stressed by clinicians.

In several experiments, we tried to find new stress situations with not only higher external validity (in the best case with face validity), but controllable and reproducible as well - a desideratum for 'internal validity' [Campbell and Stanley, 1973]. Clinical considerations show that most mental disorders reported by patients are induced socially or have their main symptoms in the social field (e.g. problems in marriage, between colleagues, with friends, within performance in social contexts). Is it possible to transfer these troubling everyday situations in the laboratory without losing their characteristics?

Group dynamics is one approach in this direction - another approach under the same consideration is reported by Kohnen and Krüger [1982]. They may be viewed in a double sense: first they have to produce and/or elicit a situation which is socially problematic and, secondly, they have to point out possibilities for the subjects to solve the emerging problems. Thus, the problem of evaluating the effects of group dynamics is twofold: Have group dynamics succeeded in generating a valid (i.e. real-life) situation and in giving more social competence to the patients?

Granting that group therapy has succeeded in generating valid situations, this technique may also be introduced in experiments evaluating drug reactions. Questions such as the following can be taken into account: Can a tranquillizing agent help in mastering a socially stressful situation? How does it act in situations with no social challenge and in phases of 'asocial' activities (e.g. meditation)? The following experiment is a first attempt to give an empirical answer. Its results may be viewed as a contribution to two problem areas: (a) What psychological and physiological effects does group therapy have? (b) How does a tranquillizing agent act in situations with low and high social challenge?

\section{Method}

\section{Selection of Social Situations}

The purpose of the experiment was to realize a wide variety of social situations. A concept which allows the integration of very asocial phases of self-occupation and very social phases of near interaction between subjects was found in the 'Gestalt therapy' $[$ Perls, 1976]. The following program was developed (it is subdivided in 41 parts; the number of each step is given in brackets).

The program started with an explanation of its aims (1), followed by a warming-up situation (2-6). Each group member chose a partner (2), with whom they discussed a given 
theme under the conditions of a 'controlled dialogue'. This part ended (as all further parts) with a discussion between the partners about their experiences (6). Then a meditation phase started (7-10), where subjects are instructed to concentrate on their bodies, especially on their faces (7) and on their hands (8). This part ended with a self-reflection (9) and a group discussion (10). Then, each member chose a new partner (11), with whom they searched for a place of their own in the room (12). Standing face-to-face, they looked into each other's eyes without speaking (13), then touched the hands of the partner (14), then played 'a silent game with the hands' (15), first with eyes opened, then closed (16). Thereafter they tried to explore the 'personal space' of the partner (How near am I allowed to go? 17). Phases of silent individual reflection (18), of discussion between the two partners (19) and in the whole group (20) ended this section.

In the next part all group members sat together in a circle with closed eyes, instructed to bring attention to their body feelings (21). Then all members took their neighbors by the hands $(22,23)$ and started a nonverbal communication with the partner to the left and to the right (24). The section ended with self-reflection (25) and group discussion (26). The next part started with the choice of a new partner (27). The partners sat face-to-face. Partner A caressed the face of partner B tenderly, the latter with closed eyes (28). After a phase of reflection (29) the roles are changed. Partner B now caressed (after a period of anticipation, 30) the face of partner A (31). The caressing was followed by a period of reflection (32). Thereafter both partners stood face-to-face, making contact only with the eyes (33). They were instructed that soon they have to embrace one another (34), then requested to do this (35), then to close their eyes, still remaining embraced (36). Slowly they had to withdraw themselves (37), then to talk about their experiences, first with the partner involved (38), then with the whole group (39). Situations 40 and 41 are the ending instruction.

The program tries to generate very different situations which can be clustered roughly (for purposes of the following data analysis) into four groups: (1) meditation or self-reflection as situations 7,8 and $9 ;(2)$ discussion between two or more members as situations 4,5 and 6; (3) anticipation of a social event as situations 2,11 and 22, and (4) touch as situations 14,15 and 16 .

From everyday experience, the ranking of these four groups may be regarded as a series of increasing social involvement, of decreasing social distance and thus (when situations are executed between strange persons) of increasing stress.

\section{Selection of Dependent Variables}

The effects of the program were evaluated with physiologically and psychologically dependent variables. For all group members, the ECG, transmitted by telemetry (system MONITEL from Messerschmidt-Boelkow, FRG) and recorded by a polygraph, was measured continuously during the whole program. For each person location and dispersion of pulse frequency were calculated, the former as difference to a resting pulse measured before the group session started.

Simultaneous scaling of psychological variables during the program was impossible without disturbing the character of the experiment as a real challenging situation. Thus, the group members received the whole text of the instruction for retrospective scaling after the end of the program, subdivided into the 41 parts mentioned above. Three dimensions were scaled for each of the 41 situations: (1) the experienced psychic strain, the scale ranging from 0 to point 50 was previously anchored at its ends - as suggested by Bartenwerfer 
[1963]; (2) the extension of perceived self-security, the scale ranged from -5 (very low) to +5 (very high), and (3) the evaluation of each single situation, the scale ranged from -5 (very negative) to +5 (very positive).

\section{Selection of the Drug}

As mentioned above, the effects of a tranquilizing agent should be evaluated. The drug used was a benzodiazepine (prazepam: a 7-chlor-1-[cyclogropyl-methyl]-1,3-dihydro5-phenyl-2H-1,4-benzodiazepine-2-on, trade-name Demetrin from Goedecke (FRG)) in dosages of 10 and $20 \mathrm{mg}$ compared to a placebo.

\section{Subjects and Procedure}

The considerations above lead to a $2 \times 3 \times 41$ experimental design with the factors sex ( 2 levels), medication ( 3 levels), and situation ( 41 levels), the latter as a repeated measurement factor. Subjects were 24 healthy, young volunteers (most of them students) aged 20-24, randomly subdivided into four experimental groups of 6 subjects each ( 3 male, 3 female). They received previous information that they would take part in a group dynamic session and that they had to take a psychotropic drug. They were paid 30 marks. All subjects in each of the four groups were not acquainted with one another. In the first section of the experiment, the ECG electrodes were applied and the resting pulse was measured. Then the subjects took the drug per os (subjects and drug randomized) in a double-blind manner. After $2 \mathrm{~h}$ of waiting (subjects could occupy themselves), the group therapy program was started in a room containing 6 chairs only. The beginning was at 4 p.m. regularly. One experimenter, introduced as a 'therapist', read the instruction text. The program lasted $90 \mathrm{~min}$. Thereafter the subjects received the instruction text for scaling (see 'Selection of dependent variables' above).

\section{Results}

The data may be viewed in two ways: (a) what effects are produced by the varying situations, and (b) what effects are produced by medication? The questions are answered by a $2 \times 3 \times 4$ ANOVA sex $\times$ medication $\times$ situation with repeated measurement on the latter factor. For analysis purposes we used the grouping of the situations in the four clusters mentioned in the section on 'Selection of dependent variables'. Table I shows the significant $F$ values referring to the four dependent variables strain, evaluation, self-security and pulse rate.

The highest $F$ values were observed on the factor 'situation'. In the observation points evaluation and pulse rate, these main effects are overlaid by an interaction with sex, i.e., situations have different effects on males and females. Since there are no interactions between medication and situation (which would mean that medication changes the experience of the situation), we arrange the following presentation under two separate headings. 


\section{The Effects of the Situation}

Figure 1 shows the variation of the four observation points depending on situations and sex. Psychic strain increased for both sexes from meditation to touching; in females, the increase was smaller in total (main effect sex; table I). Evaluation and self-security show an interaction: (a) in males, positive evaluation increased from meditation over discussion and anticipation to touch, females reported social near situations to be less positive, and (b) in males, self-security was high in meditation and touching, lower in discussion and anticipation. In females, self-security decreased with decreasing social distance.

Results $\mathbf{a}$ and $\mathbf{b}$ indicate that the program is more emotionally disturbing for women, a fact which Kohnen and Krüger (1981) interpreted as 'differential effects of stressors'. A smaller interaction is found in pulse rates. Meditation resulted in a remarkable lowering of pulse: males and females had pulse frequencies of about 12 beats/min below their resting pulse.

The absolute height of this difference must be interpreted cautiously. As figure 1 shows, pulse rate was for all groups of situations below the resting pulse (not for all 31 situations; fig. 2). That seems plausible, considering the subjects to be in a higher activation state when measured the first time (before the experiment) because they had no information except that

Table I. ANOVA sex $X$ medication $\times$ situation with the four parameters psychic strain, evaluation, security and pulse frequency

\begin{tabular}{lllll}
\hline & Strain & Evaluation & Security & Pulse \\
\hline Main effects & & & & \\
Sex & $9.71^{*}$ & - & - & - \\
Medication & - & - & - & 3.76 \\
Situation & $34.19^{*}$ & - & $4.25^{*}$ & $17.72^{*}$ \\
& & & & \\
Interactions & - & - & - & - \\
Sex $\times$ medication & - & $6.09^{*}$ & - & 3.44 \\
Sex $\times$ situation & - & - & - & - \\
Medication $\times$ situation & 2.43 & - & - & - \\
Sex $\times$ medication $\times$ situation & & &
\end{tabular}

*p smaller than $1 \%$. 


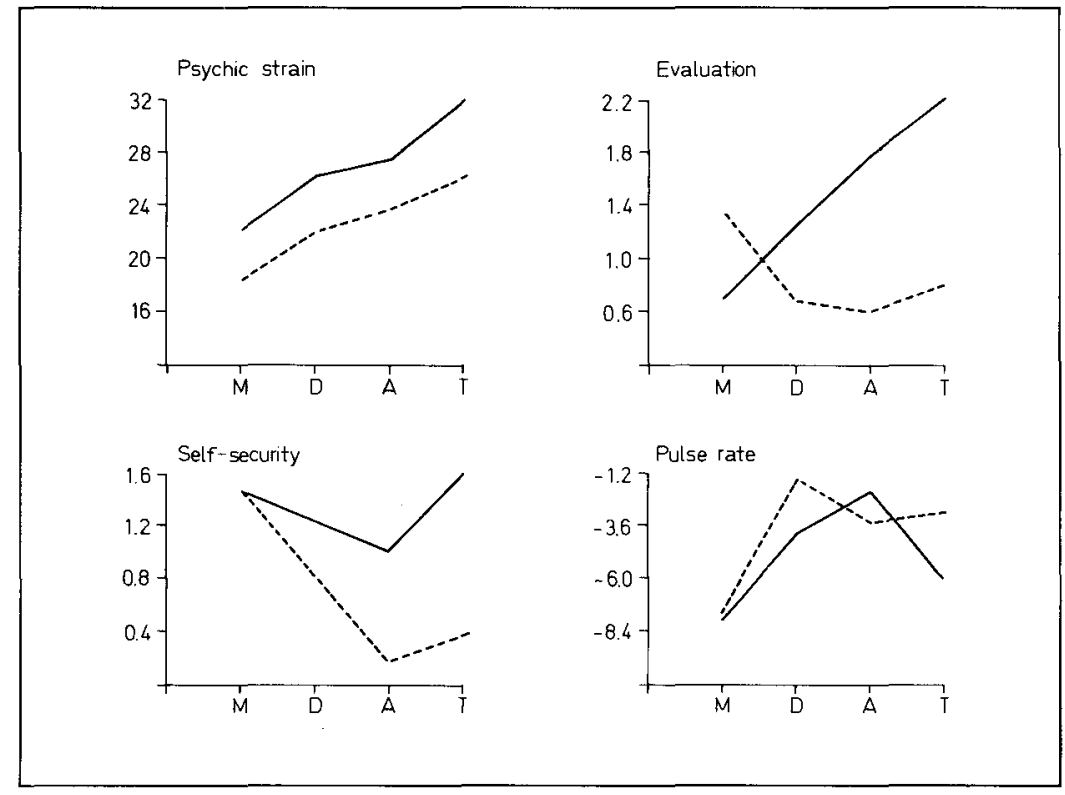

Fig. 1. Variations of the depended variables referring to the four situation types. $M=$ Meditation; $\mathrm{D}=$ discussion; $\mathrm{A}$ = anticipation; $\mathrm{T}=$ touching.

there would be 'group dynamics'. Therefore the following interpretations should be regarded only as interpretations of a relative change of pulse rates.

Pulse rate increased in discussion phases, and was also heightened in the expectancy phases. But then a very unexpected result occurs: pulse rate decreased in males when they were touching, in females it was lowered too, compared to the discussion situation. This result is surprising: we have seen psychic strain to be highest in the touch situation for both sexes. The effect of increasing strain with decreasing pulse at the same time was replicated in two other experiments by the authors [Krüger and Kohnen, 1981]. Its strangeness justifies having a closer look at its conditions. In figure 2 grand totals of all four observation points for the 41 situations are graphed (without respect to the factors sex and medication, which seems allowed regarding the high $F$ values).

There are five touch situations in the program: $14,23,28,31$, and 35 . Inspection of pulse rate (upper curve) shows that situations 28 and 31 were responsible for the 'touch effect'. In these situations subjects had to caress 


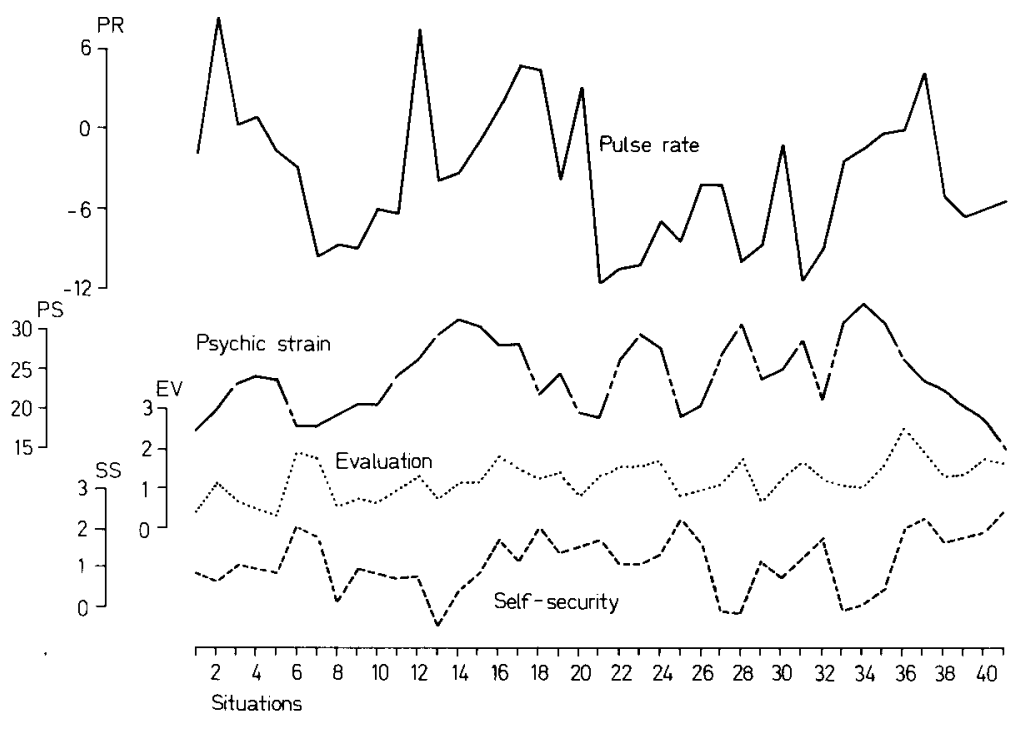

Fig. 2. Variations of the four dependent variables in the 41 situations of group therapy. $\mathrm{PR}=$ Pulse rate; $\mathrm{PS}=$ psychic strain; $\mathrm{EV}=$ evaluation; $\mathrm{SS}=$ self-security.

the face of a heterosexual partner. Psychic strain increased rapidly (dotted line below pulse), but pulse rate decreased. This asymmetry between psychic and somatic reaction has not been reported up to now in the literature, but seems very important to understand the effects of social behavior. Is it possible (to give one idea for interpretation) that, in men, there exist very early ontogenetic somatic processes, induced by social stimulation by another person, which are independent from the cognitive coping processes ontogenetic later?

A further look at figure 2 shows the drastic effects of group dynamics. Actually, it seems like an alternating bath for the subjects, both in psychic and physiologic manner. The real influence of such exercises may be expressed in some figures. (a) Psychic strain varied in subjects (regarding all 41 situations) over the total range of the scale. In the touch situation we frequently got judgements of more than 40 , indicating extreme stress (anchor point 50 was named 'fear of death in a crashing plane'). (b) Pulses individually ranged from more than 130 to 60 beats, rapid changes occurred in few minutes. 


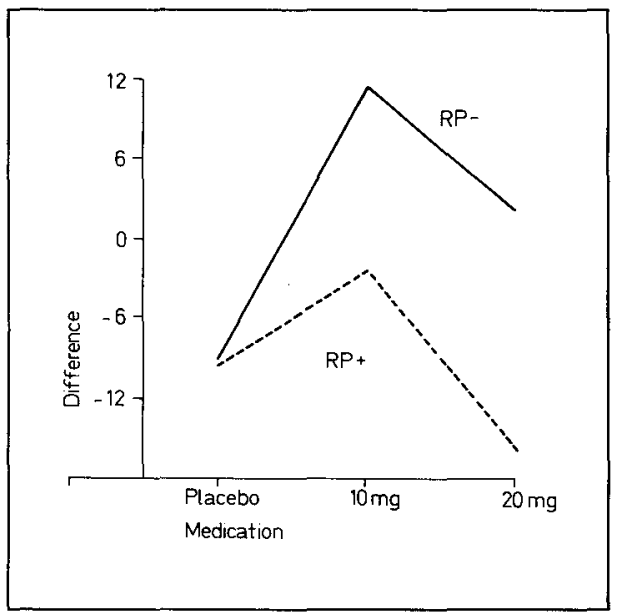

Fig. 3. Course of pulse rate (differences of situation pulse minus resting pulse) depending on dosages and separated for subjects with high and low resting pulse (RP).

The whole data show that group dynamics were able to elicit extreme reactions in subjects thus giving an important argument for the validity of our experimental situation. From a therapeutic point of view the results are of greatest interest: group dynamics are a tool really suitable to crack individual defense mechanisms and to produce real-life situations with a highly challenging character.

\section{The Effects of Medication}

As can be seen in table I, medication did not interact with the situation factor. That is a first and important result: the tranquilizing drug acts generally, not specifically. The drug-placebo differences always have the same sign, as well in high as in low strain situations (with the qualification that the present experiment only realized social situations between strange persons, e.g. no performance was required). We observed no selective drug action, which may perhaps be highly desirable for practical purposes. A tranquillizer which only acts in situations with high activation characteristics would be the ideal medication for daytime.

Because a drug-situation interaction is lacking, we omitted in our analyses of drug effects the situation variable, but introduced a new factor: the magnitude of the resting pulse. A high resting pulse before the beginning of 
the experiment may indicate a high activation level, perhaps a sort of anxiety in anticipating what will go on. If tranquillizers do not act situationspecifically (as stated above), they may act person-specifically, having different effects depending on the activation state of the organism. Therefore we subdivided the whole group at the median into subjects with high and low resting pulses. A sex $\times$ medication $X$ resting pulse ANOVA was calculated, which only yielded main effects, no interactions: (a) main effect sex (d.f.: 1,12$)$ : psychic strain $(F=7.97)$ and evaluation $(F=6.59)$ were lower in females; (b) main effect resting pulse (d.f.: 1, 12): subjects with a high resting pulse evaluated the program to be more positive $(F=4.16)$ and have a lower pulse rate in the experiment $(\mathrm{F}=9.35)$, and (c) main effect medication (d.f.: $2,12)$ : the course of pulse rate $(F=6.23)$ is shown in figure 3 , separated for subjects with high or low resting pulses.

$10 \mathrm{mg}$ prazepam led to a drastic increase of pulse rate: low resting pulse subjects had about 21 , the other group members about 8 beats/min more than subjects under placebo. Pulse rate decreased when dosage was raised. Additionally, it is remarkable that subjects with a high resting pulse had a pulse rate below their resting pulse under all medications.

First one supposes a methodological artefact: the experimental pulse is expressed as a difference to the resting pulse. For subjects with a high resting pulse, a greater amount is subtracted. The result of the placebo group was contrary to this argumentation. High and low resting pulse subjects showed the same experimental pulse here; a strong argument that the lower pulse rate of the second group was a drug effect.

\section{Discussion}

Our introductory considerations, concerning the validity of group dynamics and the suitability of such a program to evaluate tranquillizer effects in healthy subjects, were confirmed by the results. It could be shown that the program had unexpectedly high effects on the subjects both in psychological and physiological measures. The clinical inference as to these results should be: group dynamics are highly effective in producing emotional reactions (which is desirable in many cases), but this is a serious reason why they should be introduced only cautiously, (this makes group dynamics unfit for the application by amateurs). This fact was stressed by clinicians up to now - our results may be viewed as an empirical support of these warnings. 
The results indicate further that the drug acts unspecifically as to the kind of situation. A dosage effect and a dependency of drug effect from the activation state of the organism could be demonstrated. Taking all these results together, they support the assumption that group dynamics may be an efficient tool in demonstrating drug effects.

It remains to give some hypotheses about the quality of the drug effects, especially about the increased pulse rate under $10 \mathrm{mg}$ prazepam. The experiment realized situations of high privacy between strangers (touching hands, caressing the face, embracing). As pleasant as these actions can be, to do them with strangers is stressful, even for our healthy young subjects. One possible reaction is to install defense mechanisms against stressful stimulation, like blocking. Thus, for healthy subjects the stressing character of a situation may result in a reduction of the ability to react to the situation, and may lead to a slipping out of the perceived demands of the partner. In a condition like this, a low pulse rate is plausible. The tranquillizing drug acts in the direction of an unspecific easing. By lowering the base rate of activation, subjects are able to react to the demands of the situation, which now is perceived as less frightening - Kohnen and Lienert [1980] coined the term 'tranquillizer as sunglasses for the soul'. The somatic equivalent of increased ability to react may be a higher pulse rate.

This interpretation is similar to that of Lader [1978]. In his view, anxiolysis is not only decreased anxiety produced by a drug in a direct manner, but could also be an increased ability in resisting stressful events or experiences. Tranquillizers may break up defense mechanisms and thereby allow an adequate reaction to a stressful situation. Increases in pulse rates are to be expected as reference processes. An interpretation such as this demands one additional assumption: the distinction between anxiolytic and sedative effects of tranquillizers. If the dosage is too high, a clear sedation is observable in lower pulse rates and this is the reason, why the ability to react in a situation is restricted.

To get out of pure speculation: the operational analog to the 'ability to react' in our experiment, must be the dispersion of the observation points over the 41 situations. To support the hypotheses stated above, dispersion must be high in the 10-mg group, low for the placebo and 20-mg groups. In fact, all observables show this predicted course: the dispersion of psychic strain, evaluation, self-security, and pulse rate is high with $10 \mathrm{mg}$, low with placebo and $20 \mathrm{mg}$. This result is a strong argument for our interpretation. A further argument may be found in the behavior of the group of subjects with high resting pulse. We explained the high pulse rate as a consequence 
of a more neurotic anxiety of the forthcoming experiment. These high pulse rates are lowered more by medication. This differential effect is comparable with the results often found: neurotics react more strongly to medication [e.g. Janke et al., 1979].

\section{References}

Bartenwerfer, $\mathrm{H}$.: Über Art und Bedeutung der Beziehung zwischen Pulsfrequenz und skalierter psychischer Anspannung. Z. exp. angew. Psychol 10: 455-470 (1963).

Campbell, D.T.; Stanley, J.C.; Experimental and quasi-experimental designs for research (McNally, Chicago 1973).

Janke, W.; Debus, G.; Longo, N: Differential psychopharmacology of tranquilizing and sedating drugs; in Ban et al., Modern problems of pharmacopsychiatry, pp. 13-98 (Karger, Basel 1979).

Kohnen, R.; Krüger, H.-P.: Differentielle Wirkungen von Stressoren im pharmakopsychologischen Experiment. Z. Med. Psychol. (submitted, 1981).

Kohnen, R.; Krüger, H.-P.: What is a tranquilizing drug doing in verbal examinations? Int. Pharmacopsychiat. (in press, 1982).

Kohnen, R.; Lienert, G.A.: Pharmakopsychologie vs. Psychopharmakologie; in Tent, Erkennen - Wollen - Handeln (Hogrefe, Göttingen 1980).

Krüger, H.-P.; Kohnen, R.: Kognitive, emotionale und physiologische Determinanten sozialer Zustände; in Michaelis, 32. Kongress der DGfP, Zürich 1980 (Hogrefe, Göttingen 1981).

Lader, M.H.: Stress und Angstmechanismen; in Kielholz, Betablocker und Zentralnervensystem, pp. 47-52 (Huber, Bern 1978).

Perls, S.: Grundlagen der Gestalttherapie (Pfeiffer, München 1976).

Hans-Peter Krüger, Institut für Psychologie der Universität Würzburg,

Röntgenring 11, D-8700 Würzburg (FRG) 